\title{
Décadrages Décadrages
}

cinéma, à travers champs Cinéma, à travers champs

$19 \mid 2011$

Autour d'Elephant de Gus Van Sant

\section{Ludwig Van Beethoven - Gus Van Sant : vers un idéal romantique}

Musique et silence dans Elephant

\section{Gaspard Vignon}

\section{OpenEdition}

\section{Journals}

Édition électronique

URL : https://journals.openedition.org/decadrages/306

DOI : $10.4000 /$ decadrages.306

ISSN : 2297-5977

\section{Éditeur}

Association Décadrages

Édition imprimée

Date de publication : 10 octobre 2011

Pagination : $30-47$

ISBN : 978-2-9700668-3-5

ISSN : 2235-7823

\section{Référence électronique}

Gaspard Vignon, «Ludwig Van Beethoven - Gus Van Sant : vers un idéal romantique », Décadrages [En ligne], 19 | 2011, mis en ligne le 10 octobre 2012, consulté le 03 avril 2022. URL : http:// journals.openedition.org/decadrages/306 ; DOI : https://doi.org/10.4000/decadrages.306 


\title{
Ludwig Van Beethoven - Gus Van Sant :
}

\author{
vers un idéal romantique
}

Musique et silence dans Elephant

1 Nous tenons à remercier chaleureusement Alain Boillat pour sa précieuse contribution à la rédaction du présent article.

2 Nous empruntons la notion de "musique anempathique" à Michel Chion, qui précise à son propos qu'elle "nous fait voir le fond d'indifférence du monde. Dans cette mise en perspective, l'intensité émotionnelle n'est pas diminuée, au contraire, mais portée à un autre niveau " (Michel Chion, Le son au cinéma, Paris, Cahiers du cinéma, 1985, p. 125). Il précisera dans un ouvrage ultérieur: "L'indifférence de la musique est justement signifiée au spectateur pour que celui-ci l'investisse comme un miroir où se reflètent la solitude et le dérisoire de son propre destin" (Michel Chion, Un art sonore, le cinéma. Histoire, esthétique, poétique, Paris, Cahiers du cinéma, 2003, p. 384).

Dans Elephant (USA, 2003), Gus Van Sant fait le portrait d'une jeunesse tourmentée en nous présentant diverses facettes de l'adolescence. Deux d'entre elles s'opposent de façon extrême dans un même personnage, Alex, un adolescent que l'on peut supposer plongé dans une rêverie romantique lorsqu'il joue Beethoven au piano et qui, par ailleurs, deviendra le bourreau de ses camarades. Même si a priori la musique classique peut être qualifiée dans Elephant d' "anempathique», affichant une indifférence au drame, une inadéquation au contenu des images qui n'exclut cependant pas, paradoxalement, l'investissement affectif du spectateur ${ }^{2}$, nous montrerons toutefois que cette musique, langage à part entière, se fait ici l'expression de la dualité du personnage. Si l'on cherche, comme nous entendons le faire dans cette étude, à mesurer l'influence exercée par la musique sur la perception du film par le spectateur, il nous semble important de tenter de saisir quelle signification cette musique porte en elle, quelles émotions elle tend à faire naître.

Par trois fois, la musique de Ludwig van Beethoven vient clairement marquer l'esprit du spectateur. Tout d'abord, lors du parcours de Nathan, on entend en entier le premier mouvement adagio de la sonate $\mathrm{n}^{\circ} 14$ en do dièse mineur, opus $27 \mathrm{n}^{\circ} 2$ (1801). C'est une musique dont la source ne peut être identifiée dans la diégièse, qui se fait entendre de manière égale, tandis que l'univers sonore gravitant autour du personnage visualisé est quant à lui modifié en fonction du déplacement de celui-ci, allant même jusqu'à s'accorder au ralenti de la bande image. Extradiégétique, cette musique s'impose donc à nous comme un discours propre, autonome et manifeste. Dans un second temps, on assiste à une scène capitale où l'on voit Alex jouer au piano la bagatelle en la mineur Für Elise (env. 1810) ainsi que l'adagio précédemment entendu, qui se voit par conséquent rétrospectivement ancré dans le monde du 
film: cette musique qui planait sur le collège était déjà celle du tueur. Enfin, on entendra à nouveau la bagatelle en la mineur, hors récit cette fois, au cours du défilement du générique de fin et lors du plan fixe de quelques secondes qui le précède; on l'associera au personnage d'Alex, puisque le film la pose comme étant «sa» musique.

Ces morceaux sont aussi des œuvres à part entière qui préexistent au film. Le choix de la musique n'en demeure pas moins significatif, car cette dernière emporte avec elle, en plus de sa signification intrinsèque, les valeurs culturelles qui lui ont été associées. L'appréhension de la musique est certes déterminée par la présence simultanée des images, mais aussi par les autres composantes du contexte sonore. Dans Elephant, comme nous le montrerons, Gus Van Sant assigne un rôle prépondérant au silence. Ce dernier n'y est pas une absence de sons, mais, au contraire, une "musique» intérieure qui accompagne la solitude des adolescents, une atmosphère sonore intimiste dans laquelle la musique vient se fondre. C'est pourquoi nous proposons d'analyser dans un premier temps la bande sonore dans son ensemble avant de nous intéresser à la valeur propre de la musique de Beethoven, puis à la portée des interactions entre les sons et les images.

\section{La musique du silence: I'usage de sons électroacoustiques}

Elephant nous présente des "parcours" effectués par les étudiants qui déambulent dans leur lycée. Hormis les trois jeunes filles (Brittany, Jordan et Nicole), tous marchent seuls. Ainsi, leurs longues promenades sont des scènes généralement dépourvues de dialogues. On a l'impression que la caméra se contente simplement de les suivre, sans parti pris, à distance, sans s'attacher à un dialogue ou à une action spécifique. En outre, il n'y a pas non plus de musique dite "d'accompagnement» qui dominerait distinctement la bande son pour s'assurer de l'attention du spectateur. En dépit de cette distance apparente, Gus Van Sant parvient à nous placer au plus près des personnages et de leur ressenti.

S'il n'y plus de dialogues, ni même de musique clairement audible, il reste pour le spectateur les bruits environnant les adolescents et, surtout, le silence. Quand Elias photographie les deux punks dans le parc, on perçoit le souffle du vent et les mouvements des feuilles. Lorsqu'il s'éloigne après avoir pris ses clichés, on entend soudainement ce «bruit de fond" de façon très distincte, tout le reste demeurant silencieux. Le bruit devient alors par contraste l'expression du silence qui règne autour du personnage sur lequel notre attention visuelle a été focalisée. Dans le cinéma sonore, le silence "pur» - l'absence de tout son - est à la fois impossible, puisqu'il restera toujours les bruits en provenance de la salle, 
3 Notion empruntée à François Jost, L'œilcaméra. Entre film et roman, Lyon, PUL, 1987, p. 57. et non réaliste, car à moins d'être sourd, on est toujours confronté à un bruit, si infime soit-il. C'est pourquoi le silence n'est généralement pas rendu en privant le spectateur de tout son, car cette suppression totale serait perçue comme une manifestation extradiégétique. Pour mieux faire entendre le "silence diégétique» qui accompagne la solitude de ses personnages, Van Sant a voulu que les bruits de fond de son film soient "orchestrés". Il utilise à cette fin une musique concrète et électroacoustique qui, à l'instar des sons d'ambiance, «signifie» le silence en "composant» avec des bruits réels (extraits de leur réalité par l'enregistrement sur un médium physique) et en les réarrangeant dans une certaine harmonie qui ne permet pas moins à l'auditeur de saisir ces bruits pour eux-mêmes. Le réalisateur se sert de ce chaos sonore pour mieux faire entendre le silence et pour le rendre plus vraisemblable, à la manière d'un bruiteur qui utilise des sons parfois très hétéroclites pour enrichir le réalisme des bruits de la bande son.

Dans de nombreuses scènes, la musique électroacoustique est présente de manière presque imperceptible, tant elle donne l'illusion d'appartenir au son d'ambiance. Si l'on tend l'oreille, on peut toutefois entendre, mêlés aux bruits du lycée, des cris d'enfants, des bruits d'aéroport ou encore d'étranges sons d'orchestre. Parfois, le lycée devient luimême la source d'une symphonie de bruits, comme lorsque la caméra s'invite dans les cuisines de la cantine. La scène du parcours d'Elias à travers le hall illustre particulièrement bien ce procédé "d'immersion" sonore dont use Gus Van Sant: le tumulte des étudiants s'efface complètement et la bande son n'est plus qu'un bruit lointain de concert mixé avec quelques effets acoustiques indistincts, tandis que la caméra se fixe sur le visage de l'adolescent, suggérant une "auricularisation interne primaire» sur ce dernier ${ }^{3}$. En effet, la bande son ne nous fait pas entendre le brouhaha des élèves, ainsi que pourrait le capter un micro qui suivrait Elias. Ce dernier ne prête pas attention aux bruits qui l'entourent: la bande son illustre plutôt son état de rêverie. Du reste, sitôt que l'adolescent sort de son repli pour saluer un de ses camarades, le son redevient diégétique et l'on saisit les paroles prononcées. C'est précisément ce procédé, poussé à l'extrême dans ce cas, qui permet au réalisateur de nous faire partager la solitude des déambulations des jeunes gens.

Il ne s'agit donc pas seulement de nous plonger visuellement dans l'environnement des personnages, mais aussi de retrouver l'acoustique propre à ce monde. Or dans leur isolement, les adolescents sont toujours confrontés au silence. Il n'y a personne à qui parler, aucune musique à écouter: ce silence - que Van Sant rend parfois "assourdissant» - les 


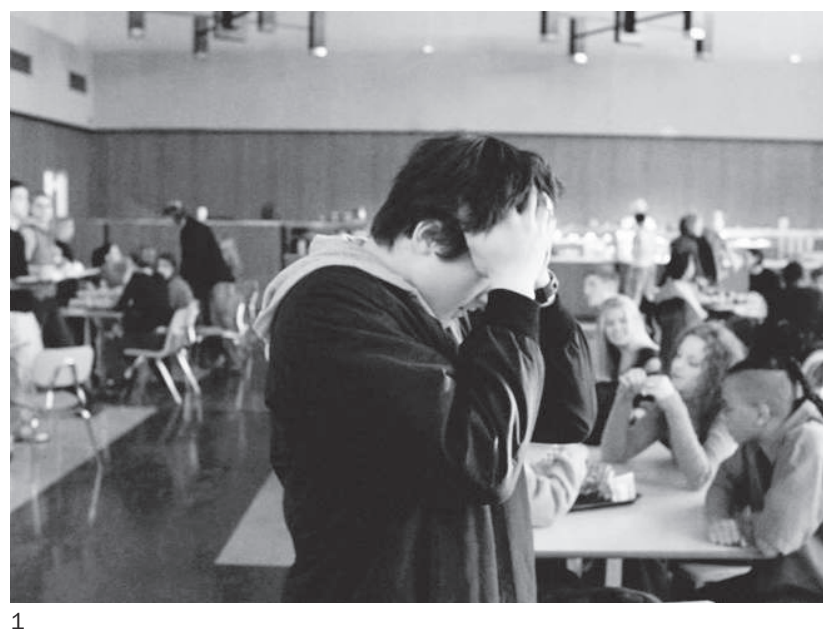

confronte à eux-mêmes ou, plutôt, confronte le spectateur à la réalité de leur condition. L'effet oppressant de ces sons est exhibé dans le film même lorsqu'Alex, dans une attitude typique de l'artiste romantique, se prend la tête dans les mains, tandis que le volume sonore s'intensifie jusqu'à devenir désagréable (fig. 1). Alex semble être le seul pour qui le brouhaha insignifiant du lycée est insupportable; il tentera de le briser par la violence. Lorsqu'il aura vidé l'établissement de tous ses bruyants occupants, un "vrai silence» se fera entendre, et, sur la bande son, des chants d'oiseaux ainsi que le doux bruit d'une rivière remplaceront la rumeur de la cohue. L'irruption de Nathan et Carrie replonge ensuite Alex dans sa folie meurtrière. Lorsque ce dernier "compte» leurs derniers instants, le silence est rendu pesant par l'ajout d'effets électroaccoustiques. Ce «silence» entendu durant tout le film est donc aussi un «silence de mort».

Sur le plan technique, la grande qualité sonore de ce silence est principalement due à l'emploi d'un MS stéréo - deux micros, un vers le haut, l'autre vers le bas - et de micros cravates pour les interprètes 4 . Il en résulte une captation efficace de la spatialisation du son, tandis que l'on reste toujours au plus près des personnages. Au début du film, par exemple, quand Elias s'éloigne de la caméra dans le parc, on entend toujours distinctement le bruit de ses pas dans les feuilles ainsi que sa respiration. De même, le réalisateur nous fait littéralement "entendre l'espace» lors de la scène du baiser, où, en plus d'un objectif grand angle qui saisit toute la solitude de John dans la pièce (fig. 2), l'effet d'écho sur les paroles renforce momentanément le sentiment de confinement et d'étouffement qui, par ailleurs, domine l'ensemble du film.
4 Alexandre Tylski, "L'oreille qui entend au loin", disponible sur le CD-Rom Elephant: un film de Gus Van Sant, Nice, Scérén/CRDP Académie de Nice, 2003. 
5 Hildegard Westerkamp est une compositrice de musique électroacoustique allemande, née en 1946. Voir www.sfu.ca/ westerka/.

6 Frances White est une compositrice américaine de musique instrumentale et électronique. Voir www.rosewhitemusic.com/.

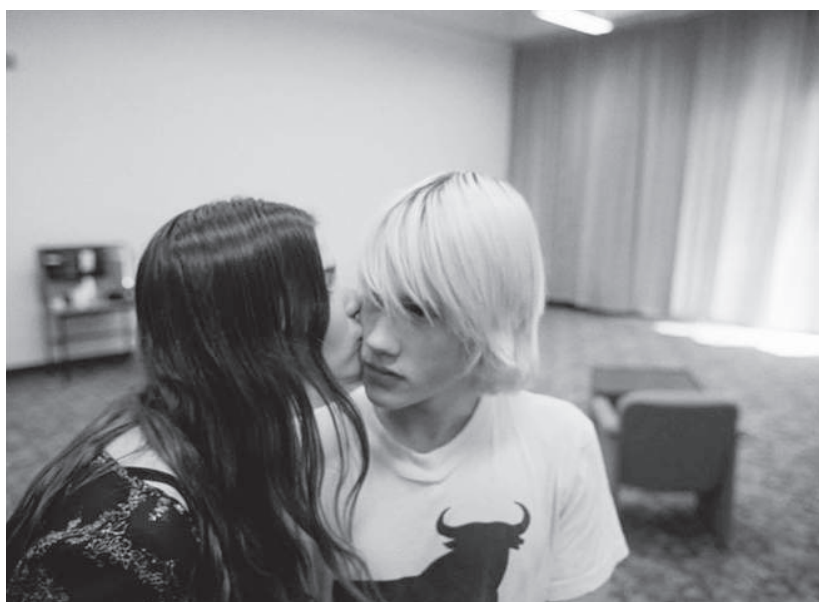

2

Dans un film qui met presqu'exclusivement en scène des adolescents, on pourrait s'attendre à ce que ce soit une musique pop propre à leur génération qui vienne accompagner leurs déboires et exprimer leur spleen existentiel. D'ailleurs, Elias et John parlent d'un concert qu'il ne faut absolument pas manquer. Or, comme on l'a vu, Gus Van Sant prend principalement pour fond sonore le «silence», dont il rend la présence vraisemblable par l'usage d'une musique électroacoustique. Bien que furtives, les manifestations de cette musique traversent l'ensemble du film et marquent en profondeur l'univers sonore de ce dernier.

Ce mélange d'occurrences sonores comprend des morceaux de musique concrète composés par Hildegard Westerkamp qui préexistent au film ${ }^{5}$ : il s'agit de Türen der Wahrnehmung (Les portes de la perception, 1989), qui se mêle à la sonate de Beethoven, notamment lors du parcours de Nathan, et de Beneath the Forest Floor (Sous le couvert forestier, 1992), qui fait entendre les chants d'oiseaux de la scène finale. S'ajoute à ces deux morceaux Walk through Resonant Landscape \#2 (Promenade à travers un paysage résonnant \#2, Frances White, 1992), qui crée une atmosphère d'étrangeté lors des déambulations de Benny et d'Eric au moment du carnage ${ }^{6}$. Comme l'indiquent déjà les titres de ces compositions, il s'agit de créer une ambiance sonore susceptible de se fondre avec l'univers visuel qu'habitent les personnages.

Le point de jonction principal entre le parcours des différents étudiants est le hall vitré dans lequel Elias croise John et le prend en photo, tandis que Michelle passe derrière eux. Cette scène est montrée trois fois, en suivant successivement chacun des personnages; l'ambiance sonore demeure identique, créant une homogénéisation qui assure une 
cohérence par-delà la fragmentation des points de vue ${ }^{7}$. La musique entendue à trois reprises est Meeting of International Conference of Technological Psychiatry, composée et interprétée par William S. Burroughs; il s'agit d'une œuvre pour orchestre symphonique dont l'orchestration n'a rien à voir avec la musique hollywoodienne habituelle. En effet, les instruments sont complètement "dilués" dans une organisation chaotique; ce n'est donc pas un hasard si ce brouhaha d'orchestre se mêle aisément, dans Elephant, aux bruits des couloirs. Si l'on tend l'oreille, on peut entendre lors de chacune des trois scènes une flûte et des notes de cor qui viennent imposer une nouvelle harmonie sonore. L'occurrence la plus remarquable de ce type de musique se situe lorsqu'Elias, dans une atmosphère de "béatitude", se rend au laboratoire photo. Il s'agit d'une sorte de cool jazz combiné à des oscillations électroacoustiques - deux morceaux sont en fait mixés ensemble ${ }^{8}$. Au final, toutes ces musiques particulières n'ont pas pour but d'offrir un espace d'évasion aux adolescents, comme le ferait une musique pop, qui se fait en général l'expression du désir de libération de la jeunesse. Au contraire, ces sons musicaux rendent compte de leur univers clos en le rendant audible, comme le fait également le "silence diégétique». Dans Elephant, le bruit de fond est élevé au rang de véritable œuvre musicale.

\section{Les sonates de Beethoven, ou le sublime accessible à tout un chacun}

De ce chaos sonore se dégage un bruit de fond plus pesant encore, plus triste, mais qui, de par sa nature profondément musicale, offre au spectateur une échappatoire hors de cet univers condamné. Il s'agit des deux morceaux pour piano de Beethoven que nous avons mentionnés ci-dessus. Hormis la scène où Alex joue les deux morceaux, chacun d'eux est entendu à une reprise avec un statut extradiégétique. Néanmoins, même dans les cas où la source n'est ni visualisée à l'écran ni suggérée dans l'univers du film, la musique de Beethoven n'est jamais la seule composante de la bande sonore. En l'occurrence, les morceaux sont systématiquement mixés avec de la musique concrète et des bruits diégétiques. De ce fait, les pièces pour piano semblent être parfaitement intégrées à l'univers sonore, d'autant que, de façon générale dans le film, la frontière entre les sons diégétiques et extradiégétiques est ténue, selon un principe de perméabilité qui affecte également la musique ${ }^{9}$. Dans l'exemple évoqué ci-dessus du parcours d'Elias se rendant au laboratoire photo, le son permet d'exprimer l'état de rêverie euphorique de ce dernier. Lors du parcours de Nathan, la musique agit de la même manière et impose son discours comme un élément à part entière de la diégèse. C'est tout le domaine subjectif du ressenti, de l'inexprimable
$7 \mathrm{~A}$ ce titre, la musique joue un rôle similaire à la mise en cadre, dont Alain Boillat montre, dans l'article du présent dossier, qu'elle produit un effet monophonique par-delà la polyphonie narrative. En ce qui concerne cette séquence montrée à trois reprises, voir fig. 26-28 de l'article de Boillat.

8 Le générique de fin mentionne la source suivante: "Supernatural infinite space (Kawabata) and Waikiki easy meat (Mano)". Ces morceaux ont été écrits et interprétés par Acid Mother Temple et The Melting Paraiso U.F.O.

9 Lorsque, dans Le son au cinéma, Michel Chion définit les trois zones d'ancrage du son par rapport à l'image (in, off et hors-champ), il met précisément l'accent sur la perméabilité de ces trois catégories (Michel Chion, Le son au cinéma, op. cit., pp. 32-44). 
10 Marcel Proust, "Journées de lecture", dans Contre Sainte-Beuve, Paris, Gallimard, 1971 [1954, posthume], pp. 162-163 [nous soulignons]. qui peut ainsi être habilement mêlé au monde réaliste et objectif qui nous est présenté par l'image. Bien entendu, Nathan ne peut entendre la musique lorsqu'il rejoint Carrie, puisque ce son est extradiégétique. Pourtant, dans la perception qu'en a le spectateur, le morceau de piano est intimement lié à la diégèse. C'est pourquoi nous avons jugé pertinent d'examiner ici en détail ce qu'expriment, en eux-mêmes, les deux morceaux de Beethoven cités par Van Sant.

Le cinéaste utilise des morceaux de musique préexistants qui apportent en effet avec eux tout le poids de la tradition, qui s'ajoute à leur discours musical. Ceci vaut en particulier pour les pièces de piano qui sont, sans aucun doute, les plus connues de Beethoven. Une telle popularité confère à ces œuvres un statut particulier. Si l'on peut reprocher au réalisateur d'avoir choisi ces pièces par manque ostensible de connaissance du répertoire, on peut aussi comprendre, au travers de ce choix, ce que doit dire la musique du monde sur lequel elle est plaquée, ou encore sur Alex, qui l'interprète.

On trouve dans Contre Sainte-Beuve de Proust une petite saynète évoquant la grand-tante de l'écrivain qui témoigne de la place qu'occupèrent, dès la fin du XIX ${ }^{\mathrm{e}}$ siècle, les sonates de Beethoven dans les foyers de la bourgeoisie aisée :

«[...] sur les choses dont les règles et les principes lui avaient été enseignés par sa mère, sur la manière de faire certains plats, de jouer les sonates de Beethoven et de recevoir avec amabilité, elle était certaine d'avoir une idée juste de la perfection et de discerner si les autres s'en rapprochaient plus ou moins. Pour les trois choses, d'ailleurs, la perfection était presque la même: c'était une sorte de simplicité dans les moyens, de sobriété et de charme. Elle repoussait avec horreur qu'on mît des épices dans les plats qui n'en exigent pas absolument, qu'on jouât avec affectation et abus de pédales, qu'en 〈recevant> on sortît d'un naturel parfait et parlât de soi avec exagération. Dès la première bouchée, aux premières notes, sur un simple billet, elle avait la prétention de savoir si elle avait affaire à une bonne cuisinière, à un vrai musicien, à une femme bien élevée. ¿Elle peut avoir plus de doigts que moi, mais elle manque de goût en jouant avec tant d'emphase cet andante si simple> [...] « Ce peut être une cuisinière très savante, mais elle ne sait pas faire le bifteck aux pommes.> Le bifteck aux pommes! Morceau de concours idéal, difficile par sa simplicité même, sorte de 〈Sonate Pathétique〉 de la cuisine.»10

Dans cette évocation "synesthésique» de ce qui fait partie d'une tradition immuable pour les gens désireux de s'élever quelque peu au-dessus 
de leur quotidien banal, Marcel Proust parvient à déterminer ce qui, dans la musique de Beethoven (et particulièrement dans les morceaux qui nous intéressent), permet de faire le lien entre une culture musicale populaire et le répertoire de la "grande musique». Elle permet à tout un chacun de combler ses aspirations artistiques grâce à la nature ambiguë d'une musique "difficile par sa simplicité même». L'adagio de la sonate et Für Elise sont des œuvres faciles d'accès, qui ne requièrent aucune virtuosité, mais qui permettent, grâce à ce qu'elles expriment, de toucher aux sentiments les plus profonds. Ainsi que l'a noté Charles Rozen, les sonates de Beethoven "possédaient autant de gravité que de passion ou d'humour. Avec elles, on était assuré de toucher au sublime»11. De cette manière, l'usage de ces deux morceaux en dit beaucoup sur les aspirations, non seulement de celui qui les joue, mais également de ceux qui vivent dans un monde profondément empreint de cette musique. C'est un choix dont la banalité découle d'un désir commun de toucher enfin au bon, au bien, au beau. A cet titre, l'utilisation de Beethoven participe de l'inscription du monde des adolescents dans une certaine "culture de masse».

\section{Une lettre d'amour: Für Elise}

La bagatelle en la mineur WoO 59, intitulée Für Elise, fait partie des œuvres dédicacées par Beethoven 12. En l'occurrence, il s'agit d'une véritable lettre d'amour, qui serait initialement dédiée à Therese Malfatti von Rohrenbach zu Dezza13, qui refusa la main du compositeur en 1810. La dédicace mal recopiée aurait renommé ladite Thérèse en Elise, mais il existe également une autre hypothèse attribuant la dédicace à une certaine Elisabeth Röckel, qui se faisait prénommer Elise ${ }^{\mathbf{1 4}}$. Laissons le débat aux spécialistes, même s'il importe d'envisager ce morceau en lien avec la vie sentimentale de Beethoven, parsemée de passions amoureuses qui furent toutes des échecs. A l'écoute de ce morceau, on comprend rapidement que l'amour, pour Beethoven, est une émotion profondément triste, nostalgique. C'est un sentiment de retour perpétuel à un état de bonheur idéal, mélancolique et serein, auquel les passions humaines ne peuvent conduire. Cet amour, pour Beethoven, est d'une beauté qui porte en elle le germe d'une infinie tristesse, car elle est un horizon inaccessible.

Für Elise est dans la tonalité de la mineur, définie comme étant «tendre et plaintive» par Marc Antoine Charpentier dans ses Règles de composition éditées en 169015. En tous les cas, le mode mineur est, même envisagé grossièrement, l'expression de la tristesse. La structure du morceau (un rondo) est composée de trois parties identiques qui sont séparées par
11 Charles Rozen, Les sonates pour piano de Beethoven, un petit guide, Paris, Gallimard, 2007, p. 20.

12 En fait, la dédicace est communément reprise pour donner un titre à l'œuvre.

13 Max Unger, "Beethoven and Therese von Malfatti ", The Musical Quarterly, vol. 11, n 1, 1925, pp. 63-72.

14 L'émission "Le mot du jour " du 9 septembre 2009 sur France musique évoque des recherches plus récentes sur l'identité de "Elise". http: //sites.radiofrance.fr/francemusique/em/ mot-jour/emission.php?e_id=70000040\&d_ $i d=395000314$ \&arch=1.

15 Pierre-Alain Clerc, Discours sur la rhétorique musicale, consultable en ligne: www.peiresc. org/Clerc.pdf, p. 47. 
deux «couplets» de caractère différent et qui comprennent chacune le thème principal (lui-même divisé en deux parties). Tout commence avec cette mélodie, que chacun reconnaîtra immédiatement, caractérisée par son début hésitant, mouvement de va-et-vient entre le mi et le ré dièse. Ce mouvement descendant puis ascendant sur une seconde mineure peut être qualifié, si l'on se fie à L'expression des intervalles de Johan Philipp Kirnberger, comme étant à la fois triste en montant et agréable en descendant16. Ici, le sentiment dominant est surtout celui de l'hésitation, d'un flottement que renforce la multiplication de ce schéma lorsque revient le thème. En outre, cette «jonglerie» entre le mi et le ré dièse est souvent le lieu de tous les effets de manche pour l'interprète peu avisé et l'on voit bien, dans Elephant, l'attention toute particulière qu'Alex porte à l'exécution de cette hésitation musicale. Puis la formule mélodique débute réellement et le ré dièse, note sensible du cinquième degré de la, redevient ré naturel. La monodie se mue alors en grands arpèges ascendants, mais la cadence parfaite nous ramène à la tonique. On a réellement le sentiment, dans cette première partie du thème, que tout se joue sur un fil. Après cette instabilité initiale, on parvient à retrouver la sérénité. De la même manière, Gus van Sant nous montre des personnages au bord du gouffre. Alex et Eric ne sont pas les seuls à avoir des problèmes, mais ce sont eux qui plongent l'univers des lycéens dans l'horreur.

Arrive ensuite la deuxième partie du thème, la mélodie s'élance - si, $d o, r e ́, m i$ - puis retombe sur le ré, puis le do, et enfin le si, dont l'aspect de dominante nous ramène au mi, hésitant, répété neuf fois de suite en sauts d'octave, pour qu'enfin l'on retrouve la plainte initiale - mi, ré dièse - répétée trois fois avant que ne recommence deux fois le thème principal, cette idée fixe que Beethoven nous fait entendre dans son habillement harmonique le plus simple. Puis arrive la première partie intermédiaire qui module en fa majeur et nous présente une mélodie guillerette, sorte d'insouciance amoureuse rompant avec la nostalgie du thème principal. A partir de là, la mélodie s'accélère, s'emballe en triples croches, et l'on rejoint la tonalité de do majeur, la joie par excellence, une joie presque artificielle, trop belle pour durer. En effet, la sensible de la mineur réapparaît et nous ramène à notre nostalgie initiale; le ré dièse refait surface et vient se frotter longuement au $m i$ avant que l'on ne retrouve la partie thématique dans sa deuxième exposition.

On passe ensuite à la deuxième partie intermédiaire, qui commence sur un grondement de double croche soutenu jusqu'à la fin de la partie. Là-dessus s'ajoutent des accords dissonants, tensions extrêmes et réso- 
lutions pathétiques, qui font de cette partie un moment de trouble, de drame. Du reste, les progressions de la basse par demi-tons accentuent cette pénibilité ambiante, cette lourdeur dramatique. Le rythme s'interrompt soudainement, puis une cadence parfaite nous amène à de grands arpèges ascendants sur plus de trois octaves et sur un rythme de triolets, rompant avec les doubles croches omniprésentes jusque-là. Cette élévation salvatrice retombe en une cascade chromatique et, inexorablement, on revient à la troisième exposition du thème, qui terminera le morceau tel qu'il a commencé. Ce grondement, avant l'instant de grâce et le retour à la nostalgie que nous expose le thème de la bagatelle, c'est celui de l'orage qui se lève sur le destin d'Alex, ce ciel qui se couvre de nuages, ainsi que Gus van Sant nous l'expose de manière brute. La grâce furtive qui s'insinue ici est similaire à celle qu'Alex semble percevoir dans un silence devenu paradisiaque avant qu'il ne tue Eric et ne replonge dans l'horreur. Enfin, en "bruit de fond", il y a cette monodie, inexorablement répétée, prenant l'allure d'un absolu, inflexible aux passions, une beauté au caractère fondamentalement inaccessible qui se teinte par conséquent d'une tristesse infinie.

\section{Seconde lettre d'amour: romance au clair de lune}

Ce fut le poète et critique musical Ludwig Rellstab qui donna à la sonate $\mathrm{n}^{\circ} 14$ en do dièse mineur, opus $27 \mathrm{n}^{\circ} 2$ le sobriquet de Mondschein-Sonate, parce que le premier mouvement lui évoquait une paisible promenade en barque sur le lac des Quatre-Cantons par un soir de pleine lune. Or, il est bien clair que cette appellation n'a pas grand-chose à voir avec l'intention originelle du compositeur, d'une part parce que cette qualification ne convient qu'au premier mouvement, d'autre part parce que, une fois encore, c'est une sonate dédiée par Beethoven à une élue de son cœur, la comtesse Giulietta Giucciardi. A l'instar de Für Elise, ce morceau constitue pour le compositeur une occasion d'évoquer par la musique son sentiment amoureux pour une femme. Ce qui va nous intéresser d'autant plus, ce sont les remarquables caractéristiques formelles qui régissent la structure générale de ce morceau et que l'on peut mettre en parallèle avec certains traits stylistiques d'Elephant. Hector Berlioz, évoquant les interprétations de cette pièce par Liszt, la décrit d'une manière brève qui suffit à dire l'essentiel:

"Il y a, dit-il, une œuvre de Beethoven connue sous le nom de la sonate en ut dièse mineur, dont l'adagio est une de ces poésies que le langage humain ne sait comment désigner: ses moyens d'action sont forts simples : la main gauche étale doucement de larges accords d'un caractère solennellement triste et dont la durée permet aux 
17 Hector Berlioz, "Concerts de M. Liszt", Journal des débats, 25 avril 1835, cité dans J. G. Prod'homme, Les sonates pour piano de Beethoven, Paris, Delagrave, 1944, p. 126.

18 Charles Rozen, op. cit., p. 146. vibrations du piano de s'éteindre graduellement sur chacun d'eux; au-dessus, les doigts inférieurs de la main droite arpègent un dessin d'accompagnement obstiné dont la forme ne varie presque pas depuis la première mesure jusqu'à la dernière, pendant que les autres doigts font entendre une sorte de lamentation, efflorescence mélodique de cette sombre harmonie.»17

On a donc deux "contraintes" fondamentales: la main gauche déroulant lentement la basse, imperturbable, et l'accompagnement de triolet, constant, "obstiné", à la manière des déambulations des adolescents dans le lycée. A cela, Beethoven ajoute une contrainte acoustique, et non des moindres puisque tout le mouvement doit être joué, sans interruption, senza sordino, à savoir sans les étouffoirs, et donc "avec pédale». Il en résulte que les cordes, une fois frappées, sont libres de sonner jusqu'à ce que le son meure de lui-même. Toutes les notes sont des plaintes qui s'éteignent dans un «bruit de fond» présent du début à la fin. De plus, le phénomène de résonance sympathique des cordes du piano provoquent l'effet suivant: les cordes qui n'ont pas été frappées entrent en résonance harmonique avec d'autres, créant "un léger brouillard, une merveilleuse sonorité éthérée» $\mathbf{1 8}$ qui n'est pas sans rappeler le "silence assourdissant» présent sur toute la bande son d'Elephant. Ces similitudes entre la forme de la sonate et la forme du film permettent ainsi, inconsciemment, une harmonie tranquille entre le parcours de Nathan, guidé par son sentiment amoureux pour Carrie, et le déroulement inflexible de cette poésie sombre et pleine d'amour. D'un côté, la caméra s'obstine à suivre le cheminement de l'adolescent et, de l'autre, "les doigts inférieurs de la main droite arpègent un dessin d'accompagnement obstiné", sorte de marche tranquille qui nous offre une isomorphie entre le vu et l'entendu. Mais les similitudes sont encore plus significatives si l'on s'intéresse à la conception des deux univers sonores du film et de l'adagio. La "sonorité éthérée» de la pièce pour piano n'est pas ici perceptible comme on pourrait le faire lors d'une écoute dédiée à la seule musique, notamment parce que, dans Elephant, le volume sonore $\mathrm{du}$ morceau est trop faible pour que nous puissions entendre le "feulement» du piano, mais également parce que les instruments du $\mathrm{XX}^{\mathrm{e}}$ siècle résonnent beaucoup et donnent ainsi trop d'ampleur au bruit de fond si l'on ne prend pas soin de caresser les cordes avec les étouffoirs. Il n'en demeure pas moins qu'il semble y avoir eu dans l'esprit de Beethoven et dans celui de Gus Van Sant une même préoccupation : faire «entendre le bruit du monde». Ainsi, par ces équivalences formelles entre musique et image, le spectateur peut aisément fondre le discours des deux médias en un seul. 
Contrairement à Für Elise, la mélodie de l'adagio ne retient pas l'attention, ne se fixe pas clairement dans la tête. Elle nage dans le flou ambiant, telle une plainte désespérée. Une note, sol dièse, s'élance et retombe brusquement sur elle-même, alors qu'une double croche brise le rythme des triolets. S'ensuit une deuxième tentative, puis enfin l'on s'élève d'une seconde mineure, jusqu'au la, et l'on revient au sol dièse, suivi de fa dièse et d'un si dont la seule fonction semble être celle de résoudre la cadence pour conclure, ainsi que l'exige la forme sonate, l'exposition du premier thème sur la dominante. Là encore, une fois arrivé sur la relative majeure, Beethoven substitue à cette dernière la tonalité mineure, et l'on replonge dans une profonde mélancolie. La même mélodie revient, sur sol bécarre cette fois, et ne sollicite que le $m i$ et le fa dièse pour conclure sur une cadence parfaite en si mineur. Le développement de la sonate poursuit cette plainte, et l'on arrive à une incroyable pédale de dominante sur dix mesures, pendant que s'égrainent toujours les triolets, imperturbables. Vient ensuite la réexposition et, dans les dernières mesures, le compositeur fait sonner le glas à la basse en lui ajoutant le rythme désespéré de la mélodie, croche pointée, double croche. Beethoven nous fait entendre une longue plainte, faite de tristesse, mais aussi d'espoir. L'arpège de mi majeur deviendra brusquement mineure et, de la même manière, si mineur deviendra plus tard si majeur. Ainsi, cet adagio agit dans Elephant de façon empathique dans la mesure où il est perçu comme une apparition prémonitoire de la tristesse générée par le massacre. Néanmoins, associé au personnage d'Alex, il semble fonctionner sur un autre registre, exprimant l'inverse de la haine inhumaine qu'on lui attribuera à la fin du film. Dès lors, il est d'autant plus intéressant de constater que Beethoven met en lien les deux extrêmes au travers de sa musique. Cette violence que nous montre la fin du film, le compositeur l'avait aussi "pressentie».

La suite de la sonate, qui n'est pas présente sur la bande son du film, se compose d'un bref allegretto en ré bémol majeur, que Liszt qualifiait de "fleur entre deux abîmes»19, fenêtre sur un bonheur idéal. De la même manière, Elephant nous montre des adolescents insouciants comme Elias ou Nathan, pris au piège entre la tristesse du monde qui les entoure et le drame qui leur sera fatal. Le dernier mouvement, presto agitato, est pareil au déchaînement d'horreur qu'est la tuerie qui clôt le film, semblable à un coup de folie: c'est le déchaînement des passions. Le contraste est immense entre le premier et le troisième mouvement. Beethoven à su joindre les extrêmes en une même œuvre; Gus van Sant a suivi le même cheminement: la sonate et le film expriment tous deux quelque

19 J. G. Prod'home, op. cit., p. 124. 
20 Gus van Sant cité par Damien Barthelemy dans Elephant: un film de Gus Van Sant [Ressource électronique], Scérén/CRDP Académie de Nice, Nice, 2003. chose d'éternel, qui dépasse le simple fait divers de la tuerie, de la haine gratuite. Dans A Clockwork Orange (Orange mécanique, USA/GB, 1971), Stanley Kubrick faisait lui aussi appel à la musique de Beethoven, mais utilisait le caractère joyeux et énergique de ses symphonies pour nous faire entendre toute la jouissance maléfique de "son" Alex, l'ultra-violent. Le personnage de Gus Van Sant, quant à lui, est bien plus introverti et, surtout, il joue lui-même l'adagio, comme s'il prenait le parti du spectateur face à la violence dont il sera l'acteur.

Lorsqu'on analyse la musique de Beethoven de cette manière, elle peut paraître destinée au film de Van Sant, comme si le réalisateur avait une conscience aiguë du discours musical. Bien entendu, il n'en est rien : le choix d'une musique relève souvent de l'intuition, outil d'analyse inconscient, mais fort efficace. Dans les faits, le réalisateur confie, en partie, la raison de ce choix musical:

«Un jour, nous sommes allés en studio pour la musique du film.

Dans la cafétéria, il y avait un piano. Alex s'y est assis et s'est mis à jouer La Lettre à Elise, l'un des seuls morceaux qu'il savait jouer. C'est là que l'idée m'est venue de lui faire jouer ce morceau dans une des scènes clés du film. J'ai donc fait mettre un piano dans sa chambre et utilisé Beethoven pour d'autres séquences du film. »20

Ainsi, le choix des morceaux découle de la volonté d'authenticité voulue par le réalisateur en laissant les acteurs être eux-mêmes, avec leurs rêves et leurs envies d'adolescents. La musique de Beethoven fait donc partie de l'identité d'Alex. Si Gus Van Sant a choisi de mettre en avant cet aspect-là de la personnalité de l'acteur, c'est que cette musique rejoint une certaine idée qu'il se fait du discours du film. Berlioz parlait d'une "de ces poésies que le langage humain ne sait comment désigner», et il en va précisément ainsi du langage musical. Ce qui a poussé le réalisateur à privilégier telle musique plutôt qu'une autre fait partie du domaine de l'intuition, cette même intuition qui détermine parfois le cadrage exact d'un plan, les coupes au montage, etc., qui se nourrit des aléas et des heureux hasards pour orienter les affects du spectateur, au-delà des mots.

\section{Des adolescents en quête d'idéal}

La musique que Gus van Sant fait surgir dans l'espace-temps habité par les lycéens est avant tout l'évocation d'un idéal. La sonate plane sur le parcours de Nathan, mais c'est toute la jeunesse qui semble hantée par le spectre de cette nostalgie amoureuse. Ce peut être un amour passionnel, pour l'adolescent au pull Lifeguard qui va rejoindre sa petite Carrie, pendant que l'on entend l'adagio. Mais pour les autres, ce sera un amour 
plus fondamental encore, le désir d'un monde meilleur. Cet ailleurs paisible, Michelle semble l'apercevoir subrepticement en levant la tête vers le ciel, vers les nuages. Elias, de son côté, transpose son idéal dans la photographie, ce sera sa seule arme de défense lorsqu'Alex et Eric font éclater la violence dans la bibliothèque. Brittany, Jordan et Nicole, quant à elles, se plaignent de tout, rien n'est assez bien dans leur vie, pas même leur amitié qu'elles remettent en question. Ce monde qui doit nourrir leurs aspirations, elles vont le vomir aux toilettes. Tous les personnages sont à la recherche d'un idéal, tel le musicien qui répète inlassablement un morceau afin d'en extraire une beauté supérieure.

$\mathrm{Au}$ début du film, on voit les nuages défiler en accéléré. Dans le même temps, la bande son nous fait entendre le "monde des hommes" par l'intermédiaire de ce qui semble être des jeunes gens qui jouent, hors-champ. Il y a dissociation entre la bande image, figurant le ciel et les nuages, ce monde idéal que certains cherchent du regard, et la bande son dont le défilement suit une temporalité humaine. Or, ces nuages sont aussi ceux de l'orage qui se lève lorsqu'Alex s'énerve devant son piano et commande ensuite des armes sur internet. Présageant la tempête qui se lèvera, la basse gronde dans l'avant-dernière partie de la bagatelle en la mineur. A la fin du film, les nuages disparaissent et font place au soleil (fig. 3-4) : c'est précisément à ce moment-là que retentit à nouveau, mêlé au chaos sonore de la musique concrète, le morceau Für Elise, désormais pareil à une longue plainte qui tente de se faire entendre parmi «le bruit du monde». Quelque chose d'éternel «flotte» au-dessus de ce monde clos. La monodie obsédante du morceau de piano sur lequel se conclut
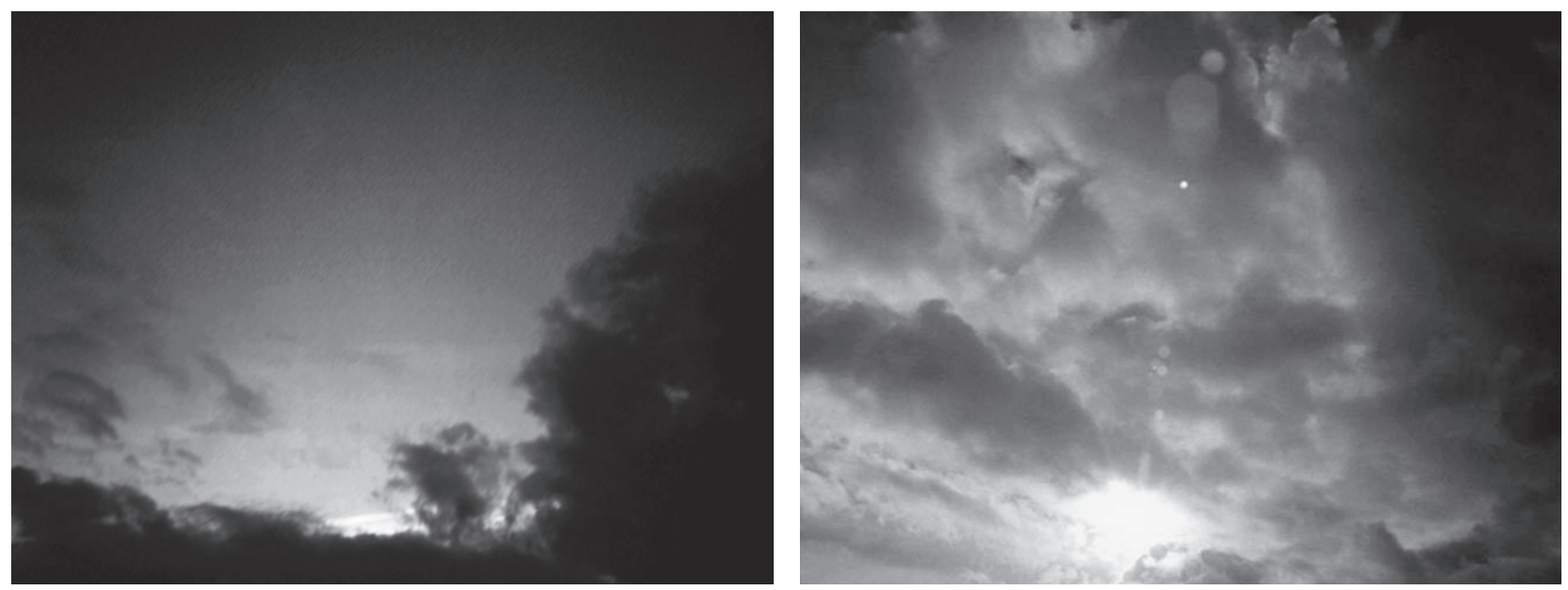
le film exprime un désir d'absolu. A la fin d'Elephant, le même morceau est perçu différemment: la musique porte désormais en elle toutes les émotions du film.

\section{Une vaine tentative d'évasion: la séquence de la chambre}

Au milieu du film, on voit Alex jouer les deux morceaux de piano que l'on connaît. Cette scène est capitale, car elle donne un ancrage diégétique à la musique et motive les apparitions "extradiégétiques» des mêmes morceaux. Les pièces de piano sont donc associées à Alex. Or, si cette musique lui plaît, c'est qu'elle exprime une émotion qui le touche (fig. 5). On est donc bien loin des adolescents fans du métal industriel de Marilyn Manson que Michael Moore interroge dans Bowling for Columbine (USA, 2002). D'ailleurs, cette musique "classique» semble également plaire à Eric, comme il le précise en entrant dans la pièce.

Tandis que la caméra suit toujours les élèves dans d'interminables travellings, elle effectue un tour sur elle-même lorsqu'elle nous montre Alex dans sa chambre. Ce dernier est comme prisonnier de cet espace, et le mouvement circulaire de la caméra vient s'ajouter à la solitude décrite par le morceau interprété par le personnage. En outre, dans son interprétation, Alex utilise abusivement la pédale, ce qui renforce sensiblement l'effet de réverbération et la suggestion de la clôture de l'espace. La caméra s'approche ensuite d'Eric et, tandis que la musique assure une continuité temporelle, on plonge dans le monde virtuel du jeu vidéo. Celui-ci est un immense espace d'errance, similaire à celui du film Gerry (Gus Van Sant, USA, 2002).

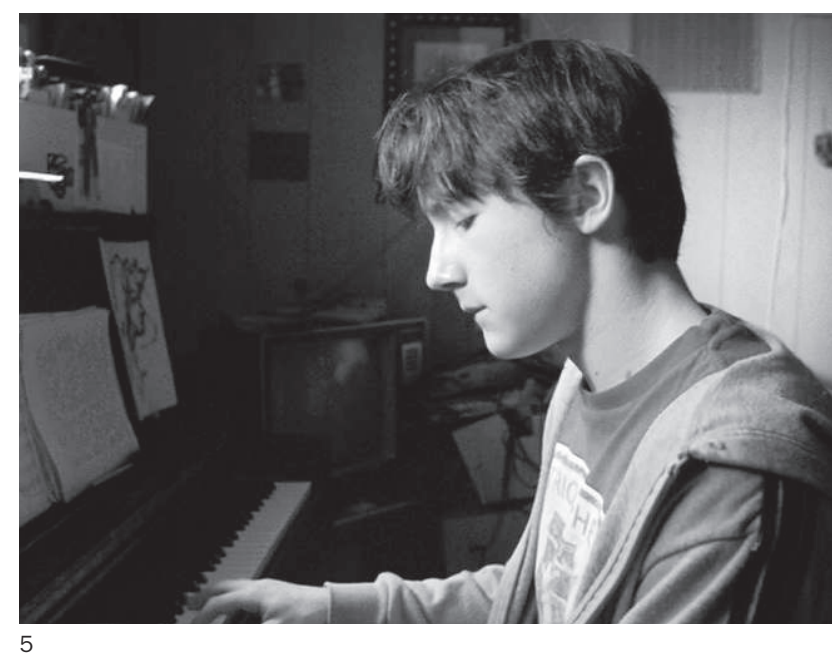


Pour Alex et Eric, la musique et le jeu vidéo offrent des espaces d'évasion. La scène est ensuite coupée par une ellipse qui laisse penser qu'Alex a continué de jouer longuement au piano, puisqu'on le retrouve alors qu'il joue la fin de l'adagio de la sonate. Mais la musique lui résiste - on le voit de dos faire un doigt d'honneur à sa partition (fig. 6) - et c'est la frustration ultime, le basculement. Alex ne peut retenir sa colère et son geste d'insulte face à la partition. Il doit reprendre le contrôle sur le monde et ce sera par la violence. C'est alors qu'on le voit acheter des armes sur internet tandis qu'Eric lit un livre. Cette musique exprime donc autant leurs aspirations que leurs frustrations.

"Jamais je n'ai vu de jour si beau et si affreux"21. Le nouveau Mal du siècle? La tuerie qui clôt le film est un événement certes prévisible, mais qui nous touche de manière totalement imprévisible. En effet, l'état de douce nostalgie qui émanait jusque-là du film est brusquement interrompu par l'objectivité des faits, et devient une sorte de mélancolie morbide. Il y a quelque chose de "beau» et d' "affreux" dans l'acte final tel qu'il est représenté chez Van Sant. La musique de Beethoven, grâce à son vaste champ d'expression et d'émotions, permet "l'union des contraires" qu'évoque Alice Laguarda dans l'article du présent dossier. Cette mélancolie de la mort relève du sublime. Alex est rongé par un mal aux tonalités romantiques qui n'est pas sans faire penser au spleen de Baudelaire et au XIX ${ }^{\mathrm{e}}$ siècle. L'auteur des Fleurs du Mal confirme dans Mon cour mis à $n u$ cette attitude contradictoire à l'égard du monde, source de toutes les tristesses: "Tout enfant, j'ai senti dans mon cœur deux sentiments
21 "So foul and fair a day I have not seen", Macbeth, Acte I, scène 3. Ce vers est cité par Alex, dans un bref soliloque prononcé au moment de la tuerie.

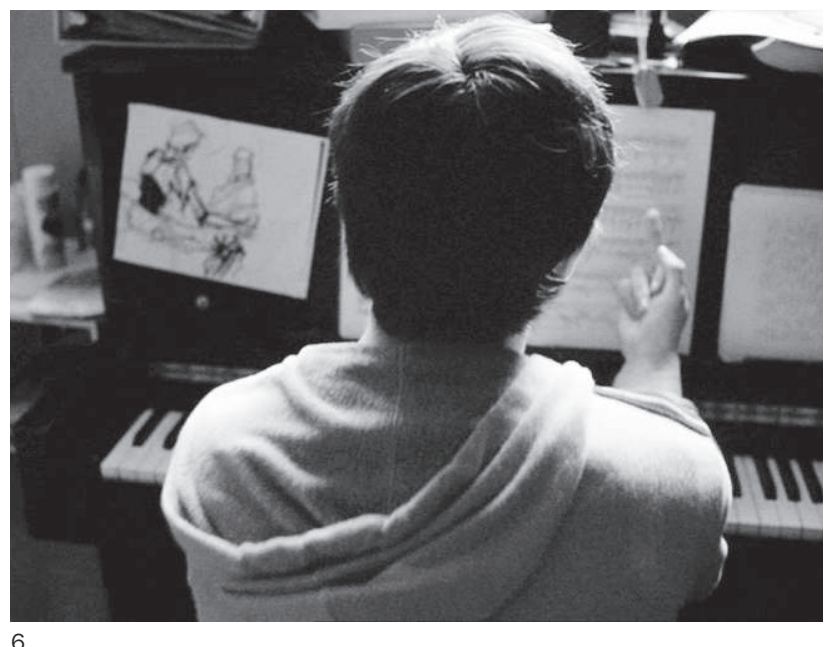


22 Charles Baudelaire, "Mon cœur mis à nu", chapitre XL, dans CEuvres complètes, tome 1 , Paris, Gallimard (coll. "La Pléiade "), p. 703.

23 Charles Baudelaire, "Spleen", dans Les Fleurs du Mal, Paris, Gallimard, 1972 [1857], p. 113. contradictoires: l'horreur de la vie et l'extase de la vie.»22 Mais surtout, succomber au spleen, c'est s'abandonner au monde, avec pour seule issue l'état de melancholia:

«Et de longs corbillards, sans tambours ni musique,

Défilent lentement dans mon âme; l'Espoir,

Vaincu, pleure, et l'Angoisse atroce, despotique,

Sur mon crâne incliné plante son drapeau noir.»23

Alex semble agir pour reprendre le contrôle. Rongé par un désespoir infini, il se rassure en devenant tout-puissant dans le monde qui l'entoure. Le paradis que l'on entend à la fin de la tuerie est, à sa manière, un "paradis artificiel» dans lequel le jeune homme se réfugie, l'espace d'un instant. Mais il est trop tard: pour s'en sortir, Alex a dû signer un pacte avec le diable et ainsi la dernière scène du film devient l'expression la plus crue de cette "union des contraires». L'adolescent ne tue plus arbitrairement, il traque ses "proies» et se permet même la cruauté ultime de choisir sa première victime. Enfin, au moment d'appuyer sur la détente, Gus Van Sant nous transporte dans un ailleurs. Tandis que la musique électroacoustique assure le continuum, le spectateur se retrouve face à un ciel nuageux dans lequel perce doucement le soleil. C'est la musique - Für Elise - qui aura le dernier mot et sa beauté, teintée d'une profonde tristesse, exprime toute "l'extase de la vie».

Si Alice Laguarda relève que la musique de Beethoven est "parodiée» dans Orange mécanique afin de mieux mettre en avant "la représentation d'un monde de l'irrévérence et de la violence gratuite", on peut dire que cet usage de la musique est aussi, dans Elephant, une preuve incontestable de l'humanité d'Alex. Quelqu'un qui est capable de comprendre et d'apprécier toute la beauté d'une telle musique, comme le font nos deux tueurs, nous paraît trop empreint de bonté pour commettre de tels actes de violence gratuite. En outre, dans Orange mécanique, c'est la musique qui permettra à Kubrick de mettre en avant l'aliénation complète qu'Alex a subie durant son traitement. Alors que la société a voulu le rendre "plus humain", elle l'a en fait insensibilisé à la beauté de Beethoven, l'a écœuré de La neuvième symphonie - l'œuvre ultime! au point qu'il se jette par la fenêtre. Il est une victime aux mains des puissants. De la même manière, l'adagio et Für Elise semblent décharger Alex de la responsabilité de son acte, non pas pour l'attribuer à la société, comme peut le faire de manière logique et rationnelle Michael Moore, mais pour rendre l'adolescent victime d'un mal plus profond encore, presque poétique. Ce n'est pas le seul lycéen à avoir des problèmes, mais il est rongé par un sentiment plus profond, car il faut que le mal-être soit total pour en arriver à formuler un tel dessein. 
Chateaubriand mettait au jour dans le personnage de René, héros du récit homonyme, ce que l'on qualifiera de Maldu Siècle, sorte de tristesse inutile qui plonge la jeunesse romantique dans une mélancolie absurde. A la fin du récit, Chateaubriand répond à ces souffrances absurdes en donnant la parole au personnage du père Souël :

"On n'est point, monsieur, un homme supérieur parce que l'on aperçoit le monde sous un jour odieux. On ne hait les hommes et la vie que faute de voir assez loin. Etendez un peu votre regard, et vous serez bientôt convaincu que tous ces maux dont vous vous plaignez sont de purs néants. » $\mathbf{2 4}$

Ainsi, l'œuvre de Gus Van Sant semble se dérouler comme un drame romantique des temps modernes. L'Alex d'Elephant pourrait être rappelé à l'ordre de la sorte. Le mal-être des adolescents n'est pas seulement lié à l'échec de la société américaine. Ce drame qui touche le lycée d'Elephant est universel et le rôle de la musique de Beethoven dans la compréhension de ce film est capital. Alex souffre, comme beaucoup d'autres, des petits tracas quotidiens. Pourtant, le plus dramatique pour lui semble être la perte d'idéal et d'absolu dans un monde froid et étouffant. Contrairement aux poètes romantiques, l'art ne lui offrira pas d'échappatoire. $\mathrm{Au}$ final, la plus grande victime est le tueur, victime de lui-même et de sa folie, mais aussi d'un manque de valeurs lui permettant d'échapper aux souffrances terrestres. Si Chateaubriand répondait au Mal du siècle en écrivant Le génie du christianisme, Gus Van Sant, quant à lui, ne donne aucune réponse à ce drame. Les personnages de Gerry, Elephant ou Last Days (USA, 2005) sont tous perdus, seuls avec leurs malheurs, que ce soit dans un désert, un labyrinthe ou encore une forêt. Leur point commun est une absence totale de repères moraux: ils sont l'antithèse de l'ermite de Chateaubriand qui trouve le bonheur dans la solitude de l'âme:

"Que faites-vous seul au fond des forêts où vous consumez vos jours, négligeant tous vos devoirs? Des saints, me direz-vous, se sont ensevelis dans les déserts? Ils y étaient avec leurs larmes, et employaient à éteindre leurs passions le temps que vous perdez peut-être à allumer les vôtres. Jeune présomptueux qui avez cru que l'homme se peut suffire à lui-même! La solitude est mauvaise à celui qui n'y vit pas avec Dieu; elle redouble les puissances de l'âme, en même temps qu'elle leur ôte tout sujet pour s'exercer. Quiconque a reçu des forces doit les consacrer au service de ses semblables; s'il les laisse inutiles, il en est d'abord puni par une secrète misère, et tôt ou tard le ciel lui envoie un châtiment effroyable.» 25
24 François René de Chateaubriand, René, Paris, Pocket, 1999 [1802], p. 129. 